\title{
Donor-impurity-related optical absorption and refractive index changes in GaAs/AlGaAs core/shell spherical quantum dots
}

\author{
Zhi-Hai Zhang ${ }^{1 *}$, Guoce Zhuang ${ }^{1}$, Kang-Xian Guo ${ }^{2}$, Jian-Hui Yuan ${ }^{3 * *}$ \\ ${ }^{1}$ School of New Energy and Electronic Engineering, Yancheng Teachers \\ University, Yancheng, 224051, China \\ ${ }^{2}$ Department of Physics, College of Physics and Electronic Engineering, Guangzhou \\ University, Guangzhou 510006, China \\ ${ }^{3}$ Department of Physics, Guangxi medical university, Nanning, Guangxi, 530021, China
}

\begin{abstract}
In this study, the linear and nonlinear intersubband optical absorption coefficients (OACs) and refractive index changes (RICs) in GaAs/AlGaAs core/shell spherical quantum dots (QDs) are theoretically investigated within the framework of the compact-density-matrix approach and iterative method for both cases with and without on-center impurity. The energy eigenvalues and their corresponding eigenfunctions of the system are calculated by using the differential method. Our results show that the position and the magnitude of the resonant peaks of the nonlinear OACs and RICs depend strongly on the hydrogenic impurity and QDs radius. It was found that the total OACs indicates saturation at sufficiently high incident intensities. Also we found that the binding energy of the ground and excited states of the impurity depend on the QDs radius.
\end{abstract}

Keywords: Optical properties; Quantum Dots; hydrogenic impurity; Binding energy.

\footnotetext{
${ }^{*}$ Corresponding author

${ }^{* *}$ Corresponding author

Email addresses: zhangzhihai3344@mail.bnu.edu.cn; Tel: 183-6114-8893 (Zhi-Hai Zhang ${ }^{1}$ ), jianhui831110@163.com; Tel:150-7882-3937 (Jian-Hui Yuan ${ }^{3}$ )
} 


\section{Introduction}

With recent rapid advances of modern technology, such as molecular beam epitaxy, metal organic chemical vapour deposition, and electron lithography, it has become possible to produce a variety of dimensionality semiconductor nanostructures $[1,2]$, which have enabled electrons to be confined in semiconductor nanostructures. Because of the confinement effects, one can expect the exotic behaviour of optical and electrical properties in these dimensionality semiconductor nanostructures, which are entirely different from their counter parts of bulk materials. Three dimensional quantum confinement of carriers in semiconductor QDs is the ability to tune their optoelectronic properties by careful design of their size, composition, shape and strain, which makes the semiconductor QDs structures so attractive. That offer a wide range of potential applications for optoelectronic devices. Some of these applications include semiconductor lasers, single-electron transistors, quantum computing, optical memories and infrared photodetectors $[3,4,5,6]$. So, it is important to study the electronic structures and optical properties of semiconductor QDs.

During last few decades, the nonlinear optical properties of low-dimensional semiconductor QDs structures have been extensively studied both experimentally and theoretically $[7,8]$. There are a few important factors that affect the linear and nonlinear optical properties, such as electric field, magnetic field, hydrogenic impurity, hydrostatic pressure and temperture $[9,10,11]$, which can tune the band gap of dimensionality semiconductor and thereby the change in property may be applied for the electro and opto-electronic devices. The presence of impurity in nanostructures influences greatly the electronic and optical properties. All semiconductor devices factually incorporate dopants as a crucial ingredient for their proper functioning. Thus understanding of the nature of impurity states in semiconductor QDs structures is one of main problems in the semiconductors low dimensional systems. Perez-Merchano et al. and Zhu et al. made the study of confinement effects on the impurity states in QDs $[12,13,14]$. M. Dezhkam et al. have investigated the effects of hydrogenic donor impurity on subband structure and nonlinear optical properties in hemispherical QD published in 2014. The obtained results show that as the dot size increases, the absorptions and dispersions increase and exhibit red shifts. Adding impurity to the system results in the blue shifts and the reduction of the absorptions and dispersions[15]. G. Rezaei et al. have theoretically studied the simultaneous influences of applied 
electric field and an on-center hydrogenic impurity on the optical properties in a multilayered spherical QD. The obtained results show that hydrogenic impurity shifts the resonant peaks of these optical properties to the higher energy regions[16]. Yuan et al. investigated the hydrogenic impurity on intersubband transitions and optical absorption properties in parabolic QD. They found the presence of the impurity can further reduce the energy level, and impurity can induce a blueshift of the peak of the OACs[17]. All of the studies mentioned above have shown that hydrogenic impurity play an important role in electronic properties of nanostructures.

Moreover, it is worth noting that due to the external factor, the calculation of energy levels and wave functions is a nontrivial task, which requires considerable theoretical effort. Due to complex realistic geometries, boundary conditions and the effects of the surrounding media, it is not possible in general to find analytical solutions using common standard procedures. To overcome this difficulty, we perform a finite-difference calculation of the electronic state, both in the absence and the presence of the donor impurity. We hope this pave the way for a more detailed description of the impurity states in the nanostructures under investigated.

In the present work, we present a numerical study of the linear and nonlinear OACs and RICs in spherical QD. In the section 2, the eigenfunctions and eigenenergies of electron states are obtained using finite difference method, and the analytical expression for the OACs and RICs are derived by means of the compact-density-matrix approach and an iterative method. In the section 3, the numerical results and discussions are presented for donorimpurity-related OACs and RICs in spherical QD. A brief summary is given in section 4.

\section{Theory}

Within the framework of effective-mass approximation, the Hamiltonian of an electron in the absence of a hydrogenic donor located at the center of a GaAs/AlGaAs core/shell spherical QD with an infinite depth potential in the region $0<r \leq R$ can be written as follows[18]:

$$
H_{0}=-\frac{\hbar^{2}}{2 m^{*} r^{2}}\left[\frac{\partial}{\partial r}\left(r^{2} \frac{\partial}{\partial r}\right)+\frac{1}{\sin \theta} \frac{\partial}{\partial \theta}\left(\sin \theta \frac{\partial}{\partial \theta}\right)+\frac{1}{\sin ^{2} \theta} \frac{\partial^{2}}{\partial \varphi^{2}}\right]-\frac{\gamma e_{s}^{2}}{\varepsilon r}
$$

where $e_{s}=e / \sqrt{4 \pi \varepsilon_{0}}$ is the reduced charge of the electron. $m^{*}$ is the effective mass of the electron in the conduction band, $\varepsilon_{0}$ and $\varepsilon$ are the permittivity of 
free space and the relative dielectric constant, respectively.

Assuming that the eigenfunctions $\psi_{n l m}(r, \theta, \varphi)=\varphi(r) Y_{l}^{m}(\theta, \varphi), Y_{l}^{m}(\theta, \varphi)$ is the normalized spherical harmonic functions; Substituting them into the Schrödinger equation $H \psi_{n l m}(r, \theta, \varphi)=E \psi_{n l m}(r, \theta, \varphi)$, we can obtain the radial functions $\varphi(r)$ that satisfy the following equation:

$$
H_{m} \varphi(r)=E_{m} \varphi(r)
$$

with

$$
H_{m}=-\frac{\hbar^{2}}{2 m^{*}}\left[\frac{\partial^{2}}{\partial r^{2}}+\frac{2}{r} \frac{\partial}{\partial r}-\frac{l(l+1)}{r^{2}}\right]-\frac{\gamma e_{s}^{2}}{\varepsilon r},
$$

where $l$ and $m$ are the quantum numbers of angular momentum and its component, respectively.

The donor eigenvalues and eigenvectors of the time-independent Schrödinger equation are calculated by the finite difference technique. This is done over a uniform mesh in the computational domain given by $0 \leq r \leq R$ with spacing $h=\frac{R}{M}$, where $\mathrm{R}$ is the dimensionless $\mathrm{QD}$ radius. The mesh is given by $r_{j}=(j-1) h$ with $1 \leq j \leq M+1$. The time-independent Schrödinger equation (Eq.(2)) in the presence of hydrogenic impurity under finite difference method can be written as follows:

$$
-\frac{\hbar^{2}}{2 m^{*}}\left[\frac{\varphi_{j+1}-2 \varphi_{j}+\varphi_{j-1}}{h^{2}}-\frac{2}{r_{j}} \frac{\varphi_{j+1}-\varphi_{j}}{h}+\frac{l(l+1)}{r_{j}^{2}} \varphi_{j}\right]-\frac{\gamma e_{s}^{2}}{\varepsilon r_{j}} \varphi_{j}=E \varphi_{j},
$$

Here, the additional boundary conditions are taken into account to discuss confined state of the hydrogenic impurity, ie, $\varphi_{1}=\varphi_{M+1}=0$. The matrix (Eq.(4)) is diagonalized to obtain the donor eigenvalues and eigenvectors of GaAs/AlGaAs core/shell spherical QD. For the energy levels of impurity states, these energy levels can be marked by a set of quantum number symbols, namely $(N, n, l, m)$, which represents principal quantum number $N$ (or radial quantum number $n$ ), the orbital quantum number $l$ and magnetic quantum number $m$. The quantum number $m \equiv 0$ in our following discussion, so the energy levels can be marked by $(N, l)$ or $(n, l)$, where $N=n+l+1$. The orbital quantum number $l=0,1,2, \cdots$ can be marked by usual notation $s, p, d, \cdots$. The donor binding energy $E_{B}(n, l)$ in a given state is defined by the difference of energy for that state in the absence of donor atom $(\gamma=0)$ and in its presence $(\gamma=1)$ of the hydrogenic impurity can be given as follows:

$$
E_{B}(n, l)=E(n, l, \gamma=0)-E(n, l, \gamma=1) .
$$


Next we will derive the OACs and RICs in spherical QD by the compactdensity-matrix method and the iterative procedure. The analytical expressions of the linear and the third-order nonlinear susceptibilities for a two-level quantum system are given as follows $[9,19,20,21]$. First, for the linear term,

$$
\varepsilon_{0} \chi^{(1)}(\omega)=\frac{N\left|M_{10}\right|^{2}}{E_{10}-\hbar \omega-i \hbar \Gamma_{12}} .
$$

For the third-order term,

$$
\begin{aligned}
\varepsilon_{0} \chi^{(3)}(\omega)= & -\frac{N\left|M_{10}\right|^{2}}{E_{10}-\hbar \omega-i \hbar \Gamma_{12}} \\
& \times\left[\frac{4\left|M_{10}\right|^{2}}{\left(E_{10}-\hbar \omega\right)^{2}+(\hbar \omega)^{2}}\right. \\
& \left.-\frac{\left(M_{11}-M_{00}\right)^{2}}{\left(E_{10}-\hbar \omega-i \hbar \Gamma_{12}\right)}\right] .
\end{aligned}
$$

The susceptibility $\chi(\omega)$ is related to the change in the refractive index as follow:

$$
\frac{\Delta n(\omega)}{n_{r}}=R e \frac{\chi(w)}{2 n_{r}^{2}},
$$

where $n_{r}$ is the refractive index. By using Eqs.(6-8), the linear and the third-order nonlinear RICs are obtained by

$$
\frac{\Delta n^{(1)}(\omega)}{n_{r}}=\frac{1}{2 n_{r}^{2} \varepsilon_{0}}\left|M_{10}\right|^{2} N\left[\frac{E_{10}-\hbar \omega}{\left(E_{10}-\hbar \omega\right)^{2}+\left(\hbar \Gamma_{12}\right)^{2}}\right]
$$

and

$$
\begin{aligned}
\frac{\Delta n^{(3)}(\omega)}{n_{r}}= & -\frac{N}{4 n_{r}^{3} \varepsilon_{0}}\left|M_{10}\right|^{2} \frac{\mu c I}{\left[\left(E_{10}-\hbar \omega\right)^{2}+\left(\hbar \Gamma_{12}\right)^{2}\right]^{2}} \\
& \times\left[4\left(E_{10}-\hbar \omega\right)\left|M_{10}\right|^{2}-\frac{\left(M_{11}-M_{00}\right)^{2}}{\left(E_{10}\right)^{2}+\left(\hbar \Gamma_{12}\right)^{2}}\left\{\left(E_{10}-\hbar \omega\right)\right.\right. \\
& \left.\left.\times\left[E_{10}\left(E_{10}-\hbar \omega\right)-\left(\hbar \Gamma_{12}\right)^{2}\right]-\left(\hbar \Gamma_{12}\right)^{2}\left(2 E_{10}-\hbar \omega\right)\right\}\right],
\end{aligned}
$$

where $N$ is the carrier density in this system, $\mu$ is the permeability of the system, $E_{i j}=E_{i}-E_{j}$ is the energy interval of two different electronic states, $M_{i j}$ is the matrix elements which is defined by $M_{i j}=\left|\left\langle\varphi_{i}|\operatorname{ercos} \theta| \varphi_{j}\right\rangle\right|(i, j=$ 
$0,1)$. The nonzero of the dipole transition moments should be satisfied the election rules $\Delta l= \pm 1$ and $\Delta m=0$. Here, we only consider the intersubband transitions between two states (namely, $|000\rangle$ and $|010\rangle$ ) in GaAs/AlGaAs core/shell spherical quantum dot. $I$ is the incident optical intensity and defined as

$$
I=2 \sqrt{\frac{\varepsilon_{R}}{\mu}}|E(\omega)|^{2}=\frac{2 n_{r}}{\mu c}|E(\omega)|^{2},
$$

where $c$ is the speed of light in free space. Therefore, the total refractive index change can be written as

$$
\frac{\Delta n(\omega)}{n_{r}}=\frac{\Delta n^{(1)}(\omega)}{n_{r}}+\frac{\Delta n^{(3)}(\omega)}{n_{r}} .
$$

In addition, the susceptibility $\chi(\omega)$ is related to the absorption coefficient $\alpha(\omega)$ by

$$
\begin{aligned}
& \alpha^{(1)}(\omega)=\omega \sqrt{\frac{\mu}{\varepsilon_{R}}} \frac{\left|M_{10}\right|^{2} N \hbar \Gamma_{12}}{\left(E_{10}-\hbar \omega\right)^{2}+\left(\hbar \Gamma_{12}\right)^{2}} . \\
& \alpha^{(3)}(\omega, I)=-\omega \sqrt{\frac{\mu}{\varepsilon_{R}}}\left(\frac{I}{2 \varepsilon_{0} n_{r} c}\right) \frac{\left|M_{10}\right|^{2} N \hbar \Gamma_{12}}{\left[\left(E_{10}-\hbar \omega\right)^{2}+\left(\hbar \Gamma_{12}\right)^{2}\right]^{2}}\left[4\left|M_{10}\right|^{2}\right. \\
&\left.-\frac{\left|M_{11}-M_{00}\right|^{2}\left[3 E_{10}^{2}-4 E_{10} \hbar \omega+\hbar^{2}\left(\omega^{2}-\Gamma_{12}^{2}\right)\right]}{E_{10}^{2}+\left(\hbar \Gamma_{12}\right)^{2}}\right] .
\end{aligned}
$$

So, the total absorption coefficient $\alpha(\omega, I)$ is given by

$$
\alpha(\omega, I)=\alpha^{(1)}(\omega)+\alpha^{(3)}(\omega)
$$

\section{Results and discussions}

In this section,the linear, the third-order nonlinear and the total OAC$\mathrm{s}$ and RICs of a hydrogenic donor impurity confined in a GaAs/AlGaAs core/shell spherical QD are calculated numerically. The parameter adopted in our calculation are as follows[11, 22]: $m^{*}=0.067 m_{0}\left(m_{0}\right.$ is the free-electron mass), $N=5 \times 10^{16} \mathrm{~cm}^{-3}, n_{r}=3.2, T_{12}=0.14 \mathrm{ps}, \Gamma_{12}=1 / T_{12}, I=0.2$ $\mathrm{MW} / \mathrm{cm}^{2}$.

The ground state $E_{00}$, the first excited state $E_{01}$ and the energy difference between ground state and the first excited state $\Delta E$ of the spherical QD 
$\operatorname{with}(\gamma=1)$ and $\operatorname{without}(\gamma=0)$ on-center impurity as a function of radius are shown in Fig.1(a). As seen, the ground and first excited states energies decrease as the radius increases for cases with and without impurity. For large values of $\mathrm{R}$ effects of confinement potential become negligible and energies approach to the energy of a free electron, which is consistent with the previous reports[9]. And the energy difference between ground and the first excited states decreases with increasing R. Moreover, It is shown that the ground state is drastically reduced for the case impurity as the dot radius increases, because of the attractive Coulomb potential of the impurity. On the other hand, the energy difference between ground state and the first excited state is a bit larger for the case impurity, relatively. The ground(blue line) and first excited state(red line) binding energy as a function of the dot radius are shown in Fig.1(b). As seen, the ground and first excited state binding energy decrease as the dot radius increases and then diminishes to a limited value. This is due to the decrease in spatial confinement between the electron and the donor impurity, which makes the effects of donor binding energy on the impurity states become negligible. Further increasing of dot radius causes sharp decreases in the binding energy to a limiting value due to non-localized charge.

In Fig.2, the linear $\alpha^{(1)}(\omega)$, the third-order nonlinear $\alpha^{(3)}(\omega)$, and the total OACs $\alpha(\omega)$ are plotted as a function of the incident photon energy(a), the resonant peak values of the linear $\alpha^{(1)}(\omega)$, the third-order nonlinear $\alpha^{(3)}(\omega)$, and the total OACs $\alpha(\omega)$ (b) are depicted versus the QD radius $R$ without $\operatorname{impurity}(\gamma=0)$. The insets represent the variation of the product term $M_{10} E_{10}$ with the QD radius. From Fig.2(a), we clearly observe that (1) the magnitude of the resonant peaks of the nonlinear absorption coefficient is significantly reduced with the increasing of $\mathrm{QD}$ radius $R$. And the magnitude of the linear absorption coefficient increases slightly. Moreover, the large nonlinear change generated by the $\chi^{(3)}$ term is opposite in sign of the linear change generated by the $\chi^{(1)}$ term. Thus the total absorption coefficient is increased by the linear term, but it is significantly reduced by the third-order nonlinear term. It is interpreted that the peak of the linear(nonlinear) term depends on $M_{10}^{2} E_{10}\left(M_{10}^{4} E_{10}\right)$. And the product term $M_{10} E_{10}$ decreases with the increasing of $\mathrm{QD}$ radius $R$ (see the insets in Fig.2(a))[9]. The nonlinear term makes a considerable contribution to the magnitude of the total OAC, so the larger the $\mathrm{QD}$ radius, the more the total OAC is reduced. It can be easily determined that the OACs as a function of QD radius $R$ have a feature similar to those reported by a number of authors[23, 24]. (2) the linear, the 
third-order nonlinear and the total OACs have a resonance peak where photon energy equal to the energy difference $E_{01}$ between ground states $E_{00}$ and first excited states $E_{01}$, which is the resonance photon energy. (3) the resonant peak positions shift toward lower energies(red shift) as the QD radius becomes larger. The physical origin is that the energy difference between the subbands will decrease with increasing QD radius. The results are in good agreement with those of Fig.1(a). (4) when the QD radius becomes smaller, the confinement potential effect on the nonlinear $\mathrm{OAC}$ is stronger as compared to the linear OAC whose results manifests into the peculiar shape in the total OAC curve. From Fig.2(b), we find that the confinement potential is decreased when the radius of QDs from $5 \mathrm{~nm}$ to $25 \mathrm{~nm}$. This effect is accompanied by the increase in the magnitude of $\alpha^{(1)}(\omega)$ and $\alpha^{(3)}(\omega)$ with $\alpha^{(3)}(\omega)$ being effected strongly as compared to $\alpha^{(1)}(\omega)$, which is in good agreement with Fig.2(a).

In Fig.3, the linear $\alpha^{(1)}(\omega)$, the third-order nonlinear $\alpha^{(3)}(\omega)$, and the total OACs $\alpha(\omega)$ are plotted as a function of the incident photon energy(a), the resonant peak values of the linear $\alpha^{(1)}(\omega)$, the third-order nonlinear $\alpha^{(3)}(\omega)$, and the total OACs $\alpha(\omega)$ (b) are depicted versus the QD radius $R$ with $\operatorname{impurity}(\gamma=1)$. The insets represent the variation of the product term $M_{10} E_{10}$ with the QD radius. From Fig.3(a), it can be clearly seen that the resonant peaks of the linear, the third-order nonlinear and the total OACs experience a slight blue-shift compared with Fig.2(a). This is because the energy difference between the ground and first excited states with the impurity is larger than that without the impurity(see Fig.1(a)). In addition, the magnitude of the resonant peaks of the linear and the third-order nonlinear OACs increase slightly when the impurity is taken into consideration. In accordance with Eq.(13) and Eq.(14), the peak value of the linear OAC $\alpha^{(1)}(\omega)$ and the nonlinear OAC $\alpha^{(3)}(\omega)$ are proportional to $\left|M_{10}\right|^{2} E_{10}$ and $\left|M_{10}\right|^{4} E_{10}$, respectively. Here $E_{10}$ is the main factor. $E_{10}$ becomes larger when the impurity is considered(see the insets in Fig.3(a)). So the enhancement is caused by the increment of energy interal between states of the QD by the donor impurity. The cusp shape in the total OAC curves also becomes more prominent with increasing QD radius. The variation of the OACs will be more clearly observed in Fig.3(b), it is obvious that the absorption coefficient peaks, both linear and nonlinear are enhanced when the impurity is considered. With the increase of the QD radius $R$, the magnitude of the resonant peaks of the total OACs decreases, which attributed to the decreasing value of $M_{10} E_{10}$ (see the insets in Fig.3(a)). This is completely in accordance 
with the results of Fig.3(a).

To illustrate the effect of incident optical intensity, in Fig.4 we draw the total OACs $\alpha(\omega)$ as a function of the photon energy for four different values of the incident optical intensity $I=0.2 \mathrm{MW} / \mathrm{cm}^{2}, I=0.5 \mathrm{MW} / \mathrm{cm}^{2}, I=0.8$ $\mathrm{MW} / \mathrm{cm}^{2}$, and $I=1.2 \mathrm{MW} / \mathrm{cm}^{2}$ with $R=10 \mathrm{~nm}$, for both cases with the on-center impurity and without the impurity. From Fig.4, It is clear that the magnitude of the resonant peak of the total OACs $\alpha(\omega)$ decreases with increasing incident optical intensity. And when $I$ exceeds a critical value, the absorption is strongly bleached at sufficiently high incident intensities, which indicates saturation, the nonlinear term causes a collapse at the center of the total absorption peak and splits it into two peaks. Furthermore, we can also observe that the peaks of the total OACs $\alpha(\omega)$ sharply diminish regardless of whether the impurity is considered. However, the magnitude of the resonant peaks of the total OACs decreases faster when the impurity is considered. Therefore, in study of the optical properties of system the nonlinear term should be taken into account, especially when the incident optical intensity is comparatively strong.

In Fig.5(a), we present the variation of the linear $\Delta n^{(1)} / n_{r}$, the third-order nonlinear $\Delta n^{(3)} / n_{r}$, and the total $\Delta n / n_{r}$ RICs as a function of photon energy without impurity $(\gamma=0)$. As can see from Fig.5(a), the linear $\Delta n^{(1)} / n_{r}$ and the total $\Delta n / n_{r}$ RICs experience a steady increase with the incident photon energy and reach maximum values. However, the nonlinear $\Delta n^{(3)} / n_{r}$ RICs decrease quickly and reach minimum values as the incident photon energy approaches the threshold energy. Moreover, the large linear change generated by the $\chi^{(1)}$ term is opposite in sign of the third-order nonlinear change generated by the $\chi^{(3)}$ term. And the total RICs is increased by the linear term, but it is significantly reduced by the third-order nonlinear term. So the resonant peak of the total RICs will be reduced. And the resonant peak of the total RICs increases by an increase in the QD radius $R$, which will behave similar to the linear term. In addition, the resonant peaks suffer an obvious red-shift as the QD radius increases. The physical origin is that the energy difference $E_{01}$ decreases with increasing QD radius(see Fig.1(a)). To look at these behavior accurately, in Fig.5(b) the resonant peak absolute value of the linear $\Delta n^{(1)} / n_{r}$, the third-order nonlinear $\Delta n^{(3)} / n_{r}$, and the total $\Delta n / n_{r}$ RICs are plotted as a function of the QD radius $R$. As we can see from the figure, as the QD radius $R$ increases, the larger the magnitude of the resonant peaks of the linear $\Delta n^{(1)} / n_{r}$, the third-order nonlinear $\Delta n^{(3)} / n_{r}$, and the total $\Delta n / n_{r}$ RICs are. As the QD radius $R$ increases, the penetration 
of the wave function is enhanced. Therefore, the magnitude of the resonant peaks of $\Delta n^{(1)} / n_{r}, \Delta n^{(3)} / n_{r}$, and $\Delta n / n_{r}$ are enhanced with increasing $R$.

Similar effects are observed for RICs in the QD which are demonstrated in Fig.6(a). We have presented the linear, third-order nonlinear and total RICs with impurity $(\gamma=1)$. As seen from this figure, the linear index increases steadily with photon energy and reaches a maximum value. This leads to normal dispersion for any frequency of incident photon. As the photon energy approaches the threshold energy, the dispersion in RICs its sign. This anomalous dispersion is found at each resonant frequency of the QD. In our case, this anomalous dispersion region shifts toward higher energy(blue shift) when the impurity is considered. Because the energy spacing becomes larger making the resonance peaks blue shift(see Fig.1(a)). In addition, to show the relation between the RICs and QD radius $R$ more clearly, we have plotted Fig.6(b), showing the variation of resonant peak absolute values of the linear $\Delta n^{(1)} / n_{r}$, the third-order nonlinear $\Delta n^{(3)} / n_{r}$, and the total $\Delta n / n_{r}$ RICs as a function of the QD radius $R$. It is observed that the magnitude of the resonant peaks of $\Delta n^{(1)} / n_{r}, \Delta n^{(3)} / n_{r}$ and $\Delta n / n_{r}$ significantly increases with increasing QD radius $R$. It is easy to explain this characteristic. As the QD radius $R$ increases, the penetration of the wave function is enhanced. Therefore, the amplitudes of $\Delta n^{(1)} / n_{r}, \Delta n^{(3)} / n_{r}$ and $\Delta n / n_{r}$ are enhanced with increasing QD radius $R$.

\section{Conclusions}

In this paper, we investigated the binding energy and nonlinear optical properties (the linear and third-order OACs and the RICs for spherical QD. We obtained the energy eigenvalues and the energy eigenfunctions by the finite different method. We found that the energy difference $E_{10}$ and the binding energy decreases by increasing QD radius $R$ for both $\gamma=0$ and $\gamma=1$. Whereas compared the case in the absence of impurity, the energy difference $E_{10}$ drastically increases when the impurity is considered, which is opposite case for the binding energy. Simultaneously, the resonant peaks of the linear, nonlinear and total OACs and RICs exhibit a blue shift for $\gamma=1$. Furthermore, the resonant peak values of the nonlinear term become large quickly with increasing QD radius $R$. So the total OACs decreases quickly, which is strong bleached at sufficiently large $R$. Moreover, the intensity of the resonant peak for the total OACs is decreased by the impurity. The effect of the QD radius $R$ on the RICs is opposite to OACs. In addition, our 
calculated results reveal that the total OACs strong depend on the incident optical intensity $I$. With the increases $I$, the total OACs sharply diminish regardless of whether the impurity is considered, and the resonant peak is bleached strongly at stronger incident optical intensity. Our theoretical results may make a contribution to experimental studies and provide a kind of approximative modeling for the practical application such as optoelectronics devices and optical communication, etc.

Acknowledgments: Project supported by the National Science Fundation of China (under Grant No. 11604289 and 61475039), the Youth Science Foundation of Guangxi Medical University in China (under Grant No. GXMUYSF201313), the Guangxi Department of Education Research Projects in China (under Grant No. KY2015LX046), and the University National Science Foundation of Jiangsu Province in China(under Grant No. 16KJB140017).

\section{References}

[1] M. Kitamura, M. Nishioka, J. Oshinowo, Y. Arakawa, Appl. Phys. Lett. 66 (1995) 3663-3665.

[2] J. Tersoff, C. Teichert, M.G. Lagally, Phys. Rev. Lett. 76 (1996) 16751678.

[3] N. Kristaedter, O.G. Schmidt, N.N. Ledentsov, D. Bimberg, V.M. Ustinov, A.Yu. Egorov, A.E. Zhukov, M.V. Maximov, P.S. Kopev, Zh.I. Alferov, Appl. Phys. Lett. 69 (1996) 1226.

[4] E. Biolatti, I. D’Amico, P. Zanardi, F. Rossi, Phys. Rev. B 65 (2002) 075306 .

[5] H.C. Liu, Opto-Electron. Rev. 11 (2003) 1.

[6] K. Imamura, Y. Sugiyama, Y. Nakata, N. Muto, N. Yokoyama, Jpn. J. Appl. Phys. 34 (1995) L1445.

[7] P. Michler, A. Kiraz, C. Becher, W.V. Schoenfeld, P.M. Petroff, L. Zhang, E. Hu, A. Imamoglu, Science 290 (2000) 2282.

[8] M.R. Panish, Science 208 (1980) 916. 
[9] K.X. Guo, Z.M. Zhang, S. Mou, B. Xiao, J. Opt. 17 (2015) 055504.

[10] R.S. Daries Bellaa, K. Navaneethakrishnan, Solid State Commun. 130 (2004) 773-776.

[11] Z.H. Zhang, Lili Zou, K.X. Guo, J.H. Yuan, Physica E 77 (2016) 90-96.

[12] N. Porras-Montenegro, S.T. Perez-Merchanco, Phys. Rev. B 46 (1992) 9780 .

[13] N. Porass-Montenegro, J. Phys. Condens. Matter. 5 (1993) A367.

[14] J. Zhu, X. Chen, Phys. Rev. B50 (1994) 4497.

[15] M. Dezhkam, A. Zakery, Physica B 443 (2014) 70-75.

[16] G. Rezaei, M.H. Tanhaei, Opt. Quant. Electron 48 (2016) 73.

[17] J.H. Yuan, Y. Zhang, M. Li, Z.H. Wu, H. Mo, Superlatt. Microstruct. 774 (2014) 1-10.

[18] G.H. Wang, Opt. Commun. 355 (2015) 1-5.

[19] R.W. Boyd, Nonlinear Optics(Academic Press, Boston, 1992).

[20] J.H. Yuan, Y. Zhang, X.X. Guo, J.J. Zhang, H. Mo, Physica. E 68 (2015) 232-238.

[21] P.K. Jha, M. Kumar, S. Lahon, S. Gumber, M. Mohan, Superlatt. Microstruct. 65 (2014) 71-78.

[22] M. Abdullah, Farah T. Mohammed Noon, Amin H. Al-Khursan, Superlatt. Microstruct. 82 (2015) 219-233.

[23] B. Al-Nashy, S.M.M. Amin, Amin H. Al-Khursan, J. Opt. Soc. Am. B 31 (2014) 1991-1996.

[24] L. Bouzaiene, H. Alamri, L. Sfaxi, H. Maaref, J. Alloy. Compd. 655 (2016) 172-177. 


\section{Figure Captions}

Figure 1 The variation of (a)the ground state, first excited state, the energy difference between ground and first excited states, and (b)the binding energy of the ground(blue line) and first(red line) excited states as a function of the QD radius $R$.

Figure 2 (a)The linear $\alpha^{(1)}(\omega)$, the third-order nonlinear $\alpha^{(3)}(\omega)$ and the total OACs $\alpha(\omega)$ versus the incident photon energy for three different values of $\mathrm{QD}$ radius $R$, (b)the resonant peak value of the linear $\alpha^{(1)}(\omega)$, the thirdorder nonlinear $\alpha^{(3)}(\omega)$ and the total OACs $\alpha(\omega)$ are depicted versus the QD radius $R$ without impurity.

Figure 3 (a)The linear $\alpha^{(1)}(\omega)$, the third-order nonlinear $\alpha^{(3)}(\omega)$ and the total OACs $\alpha(\omega)$ versus the incident photon energy for three different values of $\mathrm{QD}$ radius $R$, (b)the resonant peak value of the linear $\alpha^{(1)}(\omega)$, the thirdorder nonlinear $\alpha^{(3)}(\omega)$ and the total OACs $\alpha(\omega)$ are depicted versus the QD radius $R$ with impurity.

Figure 4 The total OACs $\alpha(\omega)$ with and without the impurity as a function of the incident photon energy with $R=10 \mathrm{~nm}$.

Figure 5 (a)the linear $\Delta n^{(1)} / n_{r}$, the third-order nonlinear $\Delta n^{(3)} / n_{r}$, and the total $\Delta n / n_{r}$ RICs as a function of the incident photon energy for three different values of the QD radius $R$, (b)the resonant peak absolute value of the linear $\Delta n^{(1)} / n_{r}$, the third-order nonlinear $\Delta n^{(3)} / n_{r}$, and the total $\Delta n / n_{r}$ RICs are plotted as a function of the QD radius $R$ without the impurity.

Figure 6 (a)the linear $\Delta n^{(1)} / n_{r}$, the third-order nonlinear $\Delta n^{(3)} / n_{r}$, and the total $\Delta n / n_{r}$ RICs as a function of the incident photon energy for three different values of the QD radius $R$, (b)the resonant peak absolute value of the linear $\Delta n^{(1)} / n_{r}$, the third-order nonlinear $\Delta n^{(3)} / n_{r}$, and the total $\Delta n / n_{r}$ RICs are plotted as a function of the QD radius $R$ with the impurity. 
Fig.1
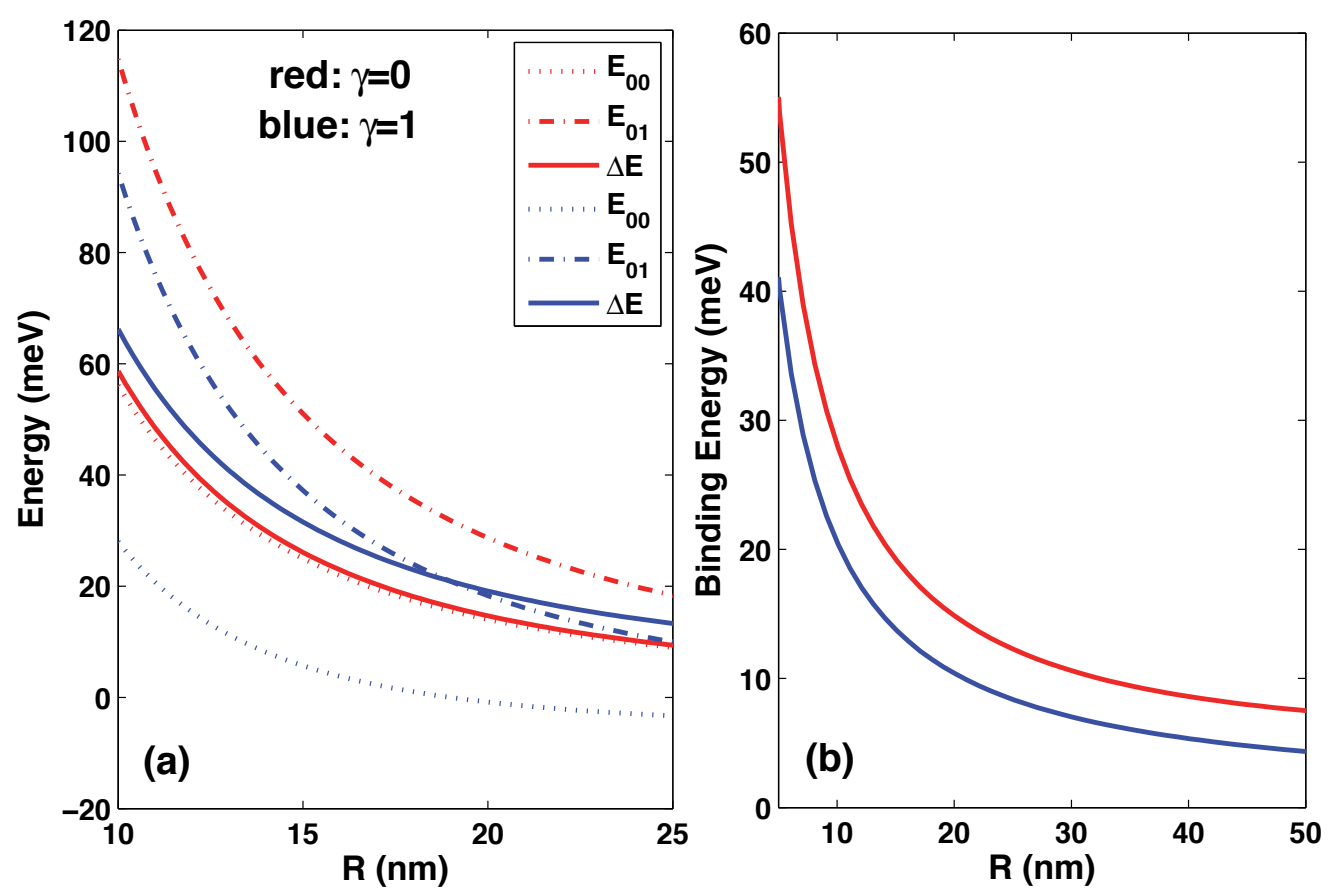
Fig.2
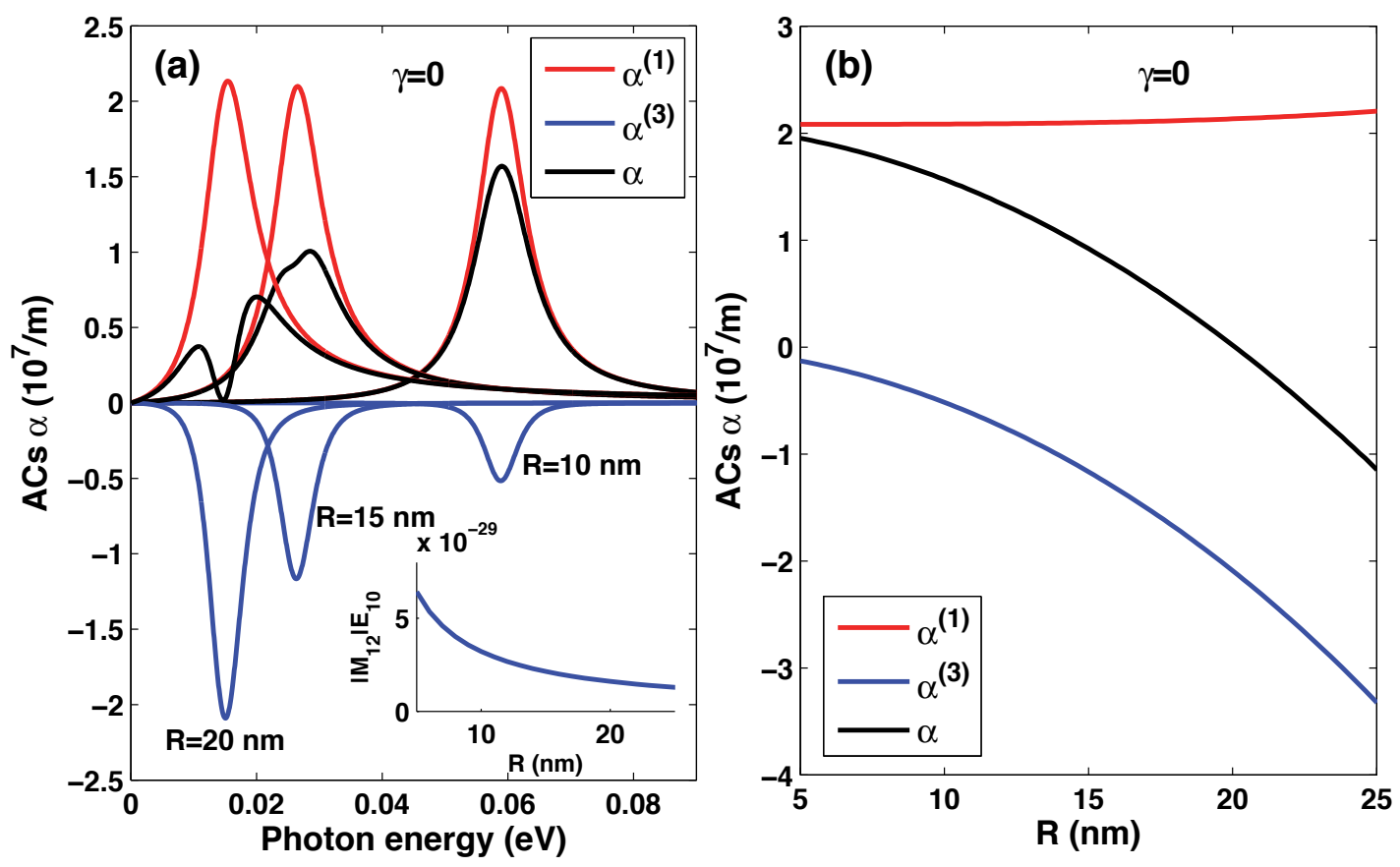
Fig.3
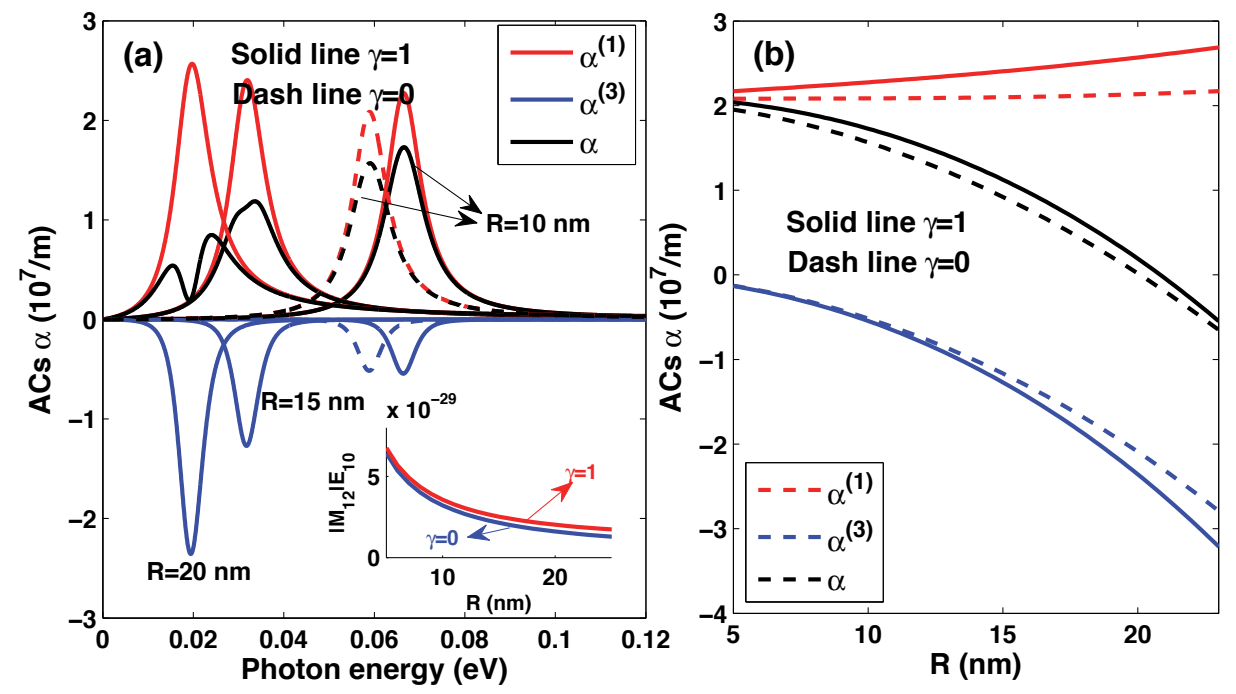
Fig.4
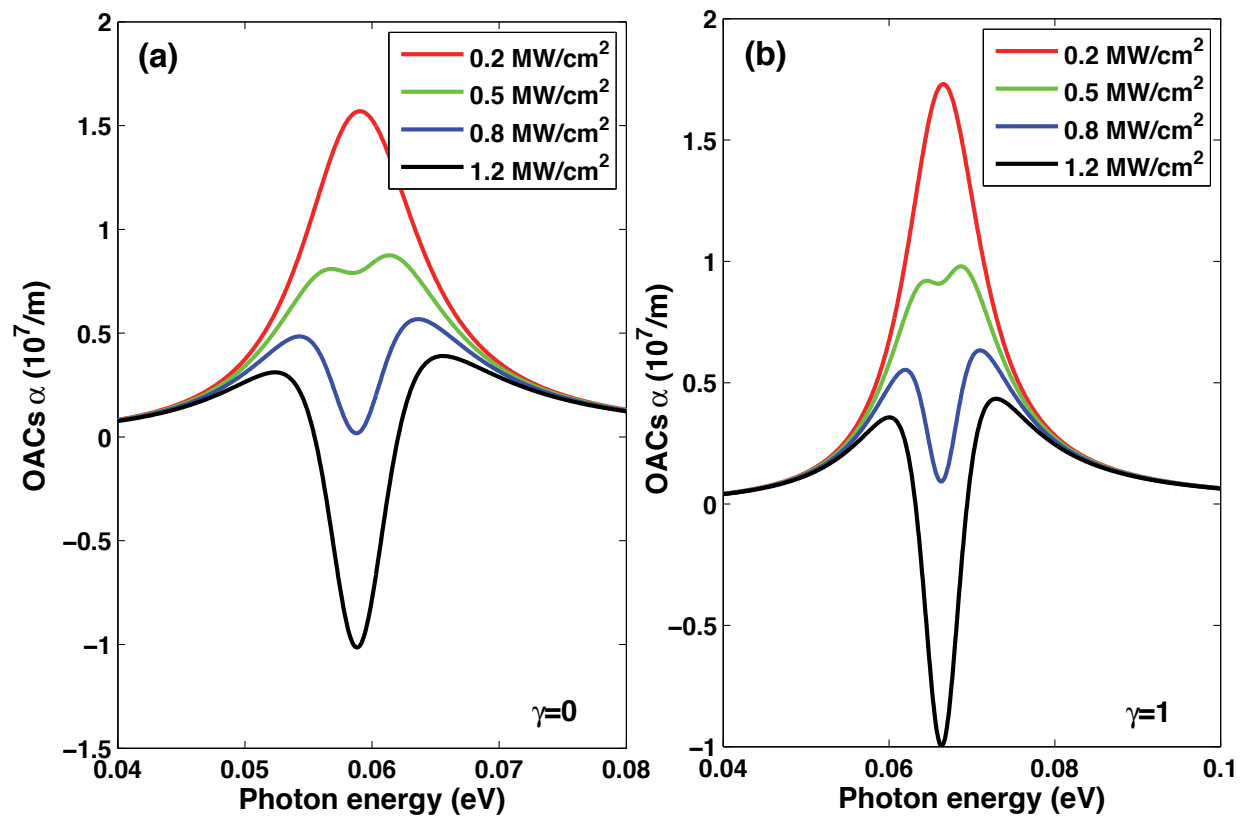
Fig.5
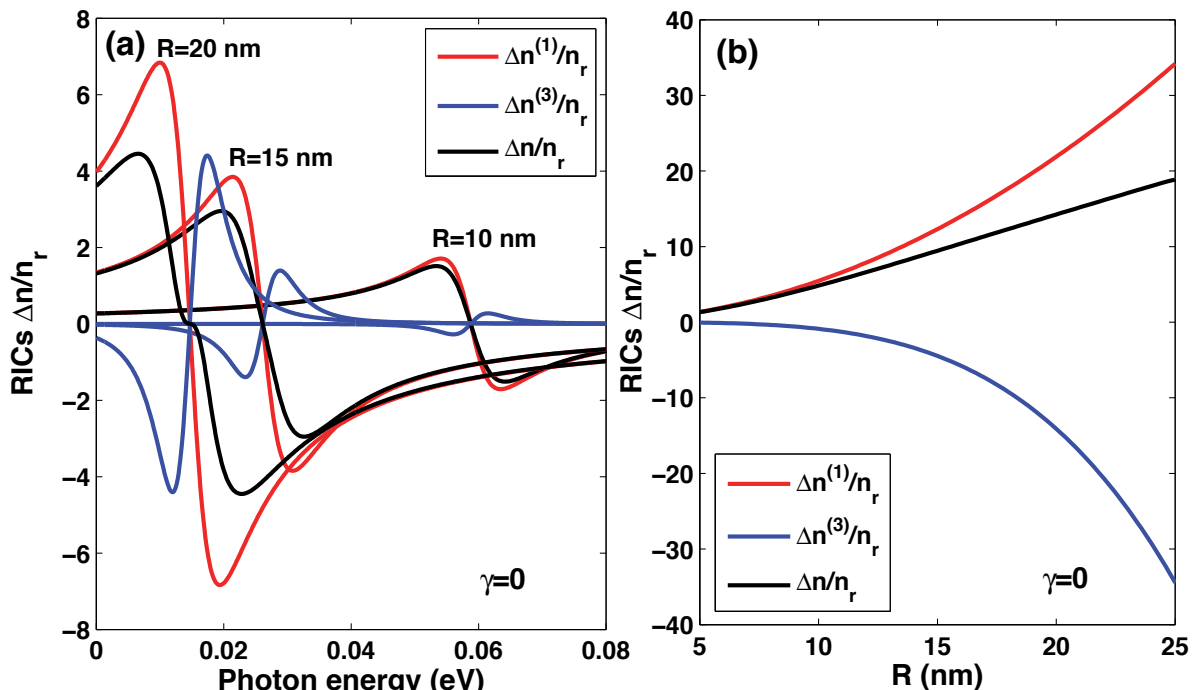
Fig.6
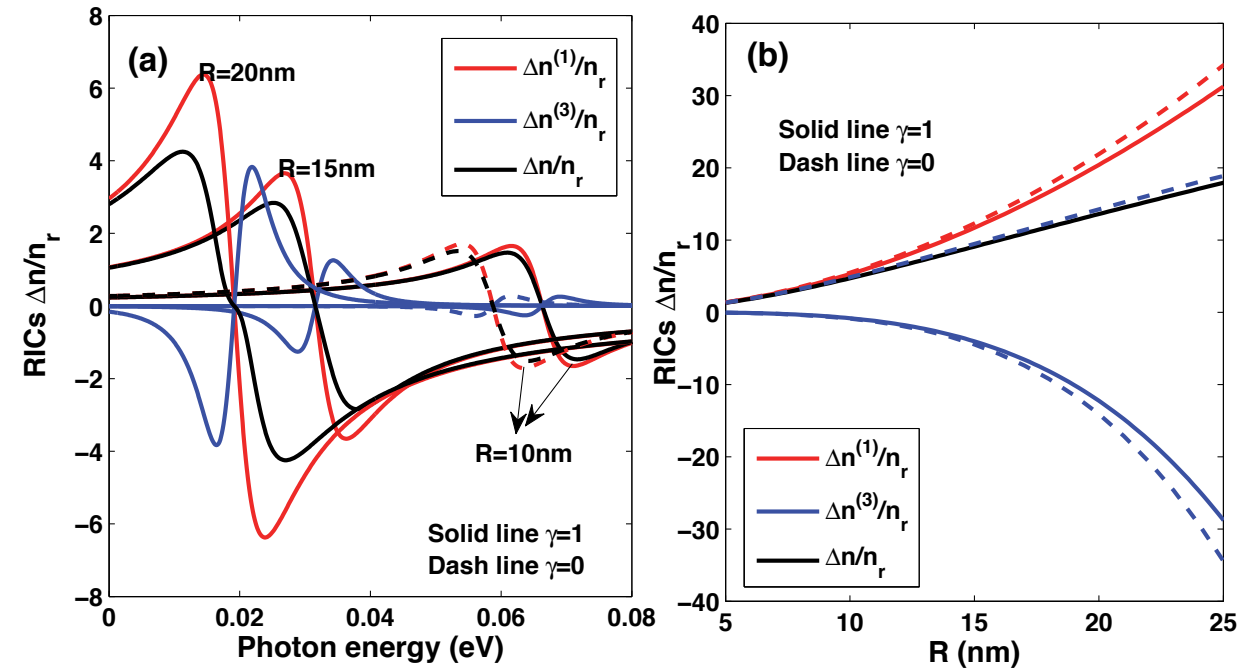